\title{
DEPENDENCIA DE LAS REDES SOCIALES VIRTUALES Y VIOLENCIA ESCOLAR EN ADOLESCENTES
}

\author{
Belén Martínez-Ferrer \\ David Moreno Ruiz \\ Departamento de Educación y Psicología Social. Universidad Pablo Olavide. \\ e-mail: bmarfer2@upo.es
}

https://doi.org/10.17060/ijodaep.2017.n1.v2.923

Fecha de Recepción: 12 Marzo 2017

Fecha de Admisión: 1 Abril 2017

\section{RESUMEN}

La evidencia empírica sugiere que la adicción a Internet se asocia con otros problemas de conducta como la sintomatología depresiva, la conducta antisocial y el cyberbullying. Sin embargo, son escasos los estudios en los que se examina la dependencia de las redes sociales virtuales (RSV) y la violencia escolar. Por ello, el propósito del presente estudio fue analizar las relaciones existentes entre la dependencia a las RSV y la violencia escolar entre iguales, en función del sexo y de la edad. Participaron 1952 adolescentes 50.4\% chicos, con edades comprendidas entre los 11 y 16 años (M edad $=14,07, \mathrm{D} \mathrm{T}=1.39$ ). Se calculó un MANOVA multifactorial. Los resultados mostraron que los adolescentes con mayor dependencia de las RSV obtuvieron las puntuaciones más elevadas en violencia manifiesta y relacional (reactiva e instrumental). Además, los chicos y las chicas con alta dependencia de las RSV mostraron una implicación similar en conductas de violencia relacional (reactiva e instrumental), mientras que los chicos con alta dependencia mostraron mayor violencia manifiesta (reactiva e instrumental). Finalmente, se discuten los resultados y sus implicaciones.

Palabras clave: dependencia, redes sociales virtuales, violencia manifiesta, violencia relacional, adolescencia

\footnotetext{
ABSTRACT

Dependence on online social networks and school violence in adolescents

The empirical evidence suggests that Internet addiction is associated with other behavioral problems such as depressive symptoms, antisocial behavior, and cyberbullying. However, few studies have examined the dependence onOnlineSocial Networks (OSN) and school violence. Therefore, the purpose of the present study was to analyze the relationships between OSN dependency and school violence among peers, considering gender and age. Participants were 1952 adolescents, $50.4 \%$ boys, aged between 11 and 16 years old ( $\mathrm{M}$ age $=14.07, \mathrm{D} \mathrm{T}=1.39$ ). A multifactorial MANOVA was
} 
calculated. Results showed that adolescents with greater dependence on OSNscored the highest on overt and relational violence (reactive and instrumental). In addition, boys and girls with high dependence on OSN showed similar involvement in relational violence (reactive and instrumental), while boys with high dependence scored the higher than girls onovertviolence (both reactive and instrumental). Finally, the results and their implications are discussed.

Key words: dependence, online social networks, overt violence, relational violence, adolescence

\section{INTRODUCCIÓN}

El uso de Internet y de las Tecnologías de la Información y la Comunicación (TICs) forman parte del estilo de vida actual en cualquier sociedad. Son numerosos los estudios que confirman el uso masivo de las TIC y de Internet entre la población joven, en especial, el uso de las Redes Sociales Virtuales (RSV) (Devine \& Lloyd, 2012; Johansson \&Götestam, 2004; Muñoz Rivas, Navarro \& Ortega, 2003). Para los adolescentes y los jóvenes, las RSV no sólo constituyen la herramienta de comunicación más frecuente, sino también, el medio para establecer y mantener nuevas relaciones interpersonales y para formar parte de grupos de amistades y grupos sociales más amplios (Echeburúa\& De Corral, 2010; Hearn\&Foth, 2007). De hecho, uno de los aspectos que resultan más atractivos de las RSV es, precisamente, la posibilidad de ser agente activo en el proceso de interacción social (Echeburúa\& De Corral, 2010).

Los datos obtenidos en distintos estudios confirmanla elevada frecuencia con la que adolescentes y jóvenes utilizan Internet y las RSV. Según el informe realizado el Instituto Nacional de Estadística (INE, 2012), el 96.5\% de los menores de 15 años acceden habitualmente a Internet.Los datos aportados por el informe europeo EU Kids Online (Garmendia, Garitaonandia, Martínez \& Casado, 2011) son convergentes con los datos del INE;el 58\% de los menores españoles utilizan Internet habitualmente.Además, el $85.1 \%$ de los adolescentes está registrado en al menos una RSV (Rial, Gómez, Braña \& Varela, 2014) y la frecuencia de uso de estas redes se sitúa en torno al 86\% (Bringué\&Sádaba, 2009; García, López de Ayala \& Catalina, 2012).Respecto al género, los datos indican que si bien los chicos utilizan más tiempo Internet (Estévez, Bayón, De la Cruz \& FernándezLiria, 2009; Muñoz-Rivas et al., 2003), las chicas afirman usar con más frecuencia las RSV (Sabater \&Bingen, 2015).

Una de las consecuencias de esta elevada frecuencia de uso de las TICs es el riesgo para sufrir algunas de las problemáticas derivadas del mal uso de las mismas (Echeburúa, Labrador \&Becoña, 2009). De hecho, la frecuencia de uso constituye una variable estrechamente vinculada con la dependencia a Internet y a las RSV. Así, en estudios recientes se ha constatado que un $11.5 \%$ de los adolescentes se conecta entre tres y cinco horas y un 5.5\% utiliza Internet más de cinco horas entre semana, porcentaje que asciende en la adolescencia tardía (Catalina García, López de Ayala \& García Jiménez, 2014). El uso problemático de Internet en España se sitúa entre el $3.7 \%$ y el $10 \%$ (Carbonell et al., 2012; Estévez et al., 2009; Muñoz-Rivas, Fernández \& Gámez-Guadix, 2010).

Actualmente, no se han establecido criterios consensuados que nos permitan diferenciar entre uso problemático, dependencia y adicción a Internet y a las RSV. Sin embargo, algunos autores han sugerido indicadores para su detección. Así, Echeburúa y De Corral (2010) han destacado criterios como los siguientes:dedicación temporal excesiva, pérdida de control y tolerancia, síntomas de abstinencia como ansiedad e irritabilidad e interferencia con las actividades de la vida cotidiana. En esta línea, Chóliz y Marco (2012) han señalado que la dependencia a Internet se caracteriza por las siguientes dimensiones: problemas en el control del impulso, preocupación obsesiva por Internet, tolerancia (uso creciente de Internet) y abstinencia, uso excesivo y problemas derivados del abuso.

En este entorno virtual, las RSVson especialmente propicias a la dependencia por aspectos 
como la facilidad de acceso, la inmediatez, la globalización, la comodidad de uso y el anonimato (Rial et al., 2014). El acceso a las RSV no solo tiene implicaciones comunicativas e influyen en las relaciones sociales que se establecen, además,pueden propiciar la creación de una falsa identidad, el distanciamientoy la distorsión del mundo real (Becoña, 2006). En este sentido, como señala Castells (2001) en la sociedad actual todo está interconectado y la realidad online y offline se entremezclan hasta configurar una totalidad.

Se ha constatado en numerosas investigaciones que el uso problemático de Internet se asocia con numerosos indicadores de problemas de ajuste psicosocial como una mayor sintomatología depresiva (Cao, Sun, Wan, Hao \& Tao, 2011; Pedrero, Rodríguez \& Ruiz, 2012), trastorno de déficit de atención con hiperactividad (Carbonell, Fúster, Chamarro \&Oberst, 2012) y disminución del rendimiento académico (Arab\& Díaz, 2015).También, se ha asociado con problemas externalizantes (Rodríguez \& Fernández, 2014) y con una mayor implicación en conductas de violencia hacia la pareja (Blanco, 2014; Martín, Pazos, Montilla \& Romero, 2016),bullying (Arnaiz, Cerezo, Gimenez\&Maquilón, 2016) cyberbullying y cybervictimización (Erdur-Baker, 2010; Giménez, Maquilón\&Arnáiz, 2015).

Como se observa de la revisión de la literatura científica, el estudio de la dependencia de Internet y sus correlatos con problemas de ajuste en el ámbito no-virtual son escasos. En este sentido, no se han encontrado investigaciones en las que se analice la relación entre la dependencia de las RSV, un ámbito de Internet utilizado, principalmente, para la interacción social, y la conducta violenta entre iguales. Por ello, el objetivo del presente estudio fue analizar las relaciones entre la dependencia de las RSV y la violencia entre iguales en adolescentes, en función del sexo y de la edad.

\section{MÉTODO}

\section{Participantes}

Participaron 1952 adolescentes (50.4\% chicos) escolarizados en nueve centros Enseñanza Secundaria Obligatoria (ESO), con edades comprendidas entre los 11 y los 16 años ( $M=14.07, D T$ = 1.39). Se establecieron dos grupos de edad correspondientes con las etapas de la adolescencia: adolescencia temprana (de 11 a 13 años) (37.3\%) y de adolescencia media (de 14 a 16) (62.7\%).

\section{Instrumentos}

Violencia escolar entre iguales. Se utilizó la Escala de Conducta Agresiva (Little, Henrich, Jones \&Hawley, 2003) (traducción bidireccional por el Grupo Lisis). Esta escala tipo Likert consta de 25 ítems que miden, con un rango de respuesta que va de 1 (nunca) a 4 (siempre) la participación en comportamientos violentos hacia el grupo de iguales. Para el presente estudio se han seleccionado las subescalas de violencia manifiesta reactiva (p. ej.. "cuando alguien me hace daño o me hiere, le pego"), violencia manifiesta instrumental (p. ej., "amenazo a otros para conseguir lo que quiero"), violencia relacional reactiva (p. ej., "cuando alguien me enfada, le trato con indiferencia o dejo de hablarle") y violencia relacional instrumental (p. ej., "para conseguir lo que quiero, desprecio a los demás"). El alpha de Cronbach de las cuatro subescalas en el presente estudio osciló entre .70 y .76 .

Dependencia de las RSV. Se utilizó la subescala de dependencia de las RSV de la escala de Socialización en la Red Virtual (e-SOC 38) desarrollada por el grupo lisis (Muñiz, 2016). Esta subescala compuesta por 8 ítems, con cuatro opciones de respuesta que van de 1(nunca) a 4 (siempre), evalúa el grado de dependencia de los adolescentes a las RSV en su interacción social (p. ej., tengo la necesidad de estar conectado/a a mis redes sociales continuamente). El alpha de Cronbach para esta subescala es de .84 . 


\section{Procedimiento}

En primer lugar, se contactó con la dirección de los centros educativos seleccionados explicando el proyecto de investigación y se concertó una entrevista para solicitar la participación en el proyecto $y$, en caso afirmativo, entregar los consentimientos informados para los progenitores y el alumnado junto con una carta explicativa de la investigación. Tras obtener los permisos, se llevó a cabo la aplicación de los instrumentos, en el que participó un grupo de investigadores expertos y entrenados. La batería de instrumentos se administró al alumnado en sus aulas habituales durante un período regular de clase donde el profesor/a-tutor/a del curso siempre estuvo presente. La participación en la investigación fue voluntaria y anónima. El estudio cumplió los valores éticos requeridos en la investigación con seres humanos, respetando los principios fundamentales incluidos en la Declaración de Helsinki, en sus actualizaciones y en las normativas.

\section{Análisis estadísticos}

Previamente al cálculo del MANOVA y de los ANOVAs se conformaron los grupos de dependencia de las RSV. Para ello, se identificaron tres grupos de adolescentes: baja dependencia de las RSV, con puntuaciones menores 0 iguales al primer cuartil, $N=467(23.9 \%)$, dependencia media de las RSV, con puntuaciones que se sitúan en el rango intercuartílico, $N=961(49.2 \%)$ y alta dependencia de las RSV, con puntuaciones iguales o superiores al tercer cuartil, $N=524(26.8 \%)$. Seguidamente, se realizó un MANOVA con el objeto de analizar las diferencias en violencia entre iguales manifiesta y relacional en función del grado de dependencia a las RSV, el sexo de los participantes y la edad. El diseño factorial fue de 3 (dependencia de las RSV bajo, medio y alto) por 2 (sexo de los adolescentes, chico o chica) por 2 (edad: de 11 a 13 y de 14 a 16 años), para examinar posibles efectos de interacción. Seguidamente, se realizaron univariadas (ANOVAS) para analizar las diferencias en las variables que resultaron estadísticamente significativas y se aplicó la prueba post-hoc de Bonferroni $(\alpha=0.05)$.

\section{RESULTADOS}

\section{Análisis descriptivos}

En la Tabla 1 se presenta la distribución de los grupos de dependencia de las RSV en función del género y del intervalo de edad. Los análisis realizados muestran una mayor proporción de chicas con alta dependencia y una mayor proporción de chicos en el grupo de baja dependencia $\left(\chi^{2}(2)\right.$ $=16.45, p<.01)$. Respecto de la edad, se ha observado una mayor proporción de adolescentes con alta dependencia a las RSV en el grupo de 14-16 años $\left(\chi^{2}(2)=45.82, p<.01\right)$.

Tabla 1.

Distribución de los adolescentes en función de la dependencia a las RSV

\begin{tabular}{|c|c|c|c|c|c|}
\hline & & & \multicolumn{3}{|c|}{ Dependencia RSV } \\
\hline & & & Baja & Media & Alta \\
\hline \multirow{4}{*}{ Sexo } & Chico & $\mathrm{N}$ & 265 & 488 & 230 \\
\hline & & $\%$ & 27 & 49.6 & 23.4 \\
\hline & Chica & $\mathrm{N}$ & 202 & 473 & 294 \\
\hline & & $\%$ & 20.8 & 48.8 & 30.3 \\
\hline \multirow{4}{*}{ Edad } & [11-13] & $\mathrm{N}$ & 228 & 353 & 147 \\
\hline & & $\%$ & 31.3 & 48.5 & 20.2 \\
\hline & [14-16] & $\mathrm{N}$ & 239 & 608 & 377 \\
\hline & & $\%$ & 19.5 & 49.7 & 30.8 \\
\hline \multirow{2}{*}{ Total } & & $\mathrm{N}$ & 467 & 961 & 524 \\
\hline & & $\%$ & 23.9 & 49.2 & 26.8 \\
\hline
\end{tabular}




\section{Análisis multivariados}

En el MANOVA calculado se encontraron diferencias estadísticamente significativas en los efectos principales de dependencia de las $R S V\left(=.904, F(8,3874)=24.98, p<.001, \eta^{2}=.050\right)$, sexo $\left(=.861, F(4,1937)=77.89, p<.01, \eta^{2}=.139\right)$. No se encontraron diferencias estadísticamente significativas en función de la edad $\left(=.996, F(4,1937)=2.18, p=.06, \eta^{2}=.004\right)$. También, se obtuvo un efecto de interacción estadísticamente significativo entre dependencia de las RSV y sexo $\left(=.991, F(8,3874)=2.09, p<.01, \eta^{2}=.004\right)$.

\section{Dependencia de las RSV}

Los resultados mostraron diferencias significativas en las cuatro dimensiones de violencia entre iguales analizadas (ver Tabla 2). Las pruebas de Bonferroni $(\alpha=.05)$ indicaron que los adolescentes con alta dependencia de las RSV obtuvieron las puntuaciones más elevadas en violencia manifiesta (reactiva e instrumental) y en violencia relacional (reactiva e instrumental), en comparación con los adolescentes con dependencia media y baja.

Tabla 2.

Medias, Desviación típicas (DT), valores Fy prueba de Bonferroni entre dependencia de las RSV, sexo de los adolescentes y violencia entre iguales

\begin{tabular}{|c|c|c|c|c|c|c|c|c|c|}
\hline & \multicolumn{5}{|c|}{ Dependencia de las RSV } & \multicolumn{4}{|l|}{ Sexo } \\
\hline & Baja & Media & Alta & $\begin{array}{l}F \\
(2,1940)\end{array}$ & $\eta 2$ & Chico & Chica & $\begin{array}{l}\mathrm{F} \\
(1,1940)\end{array}$ & $\eta 2$ \\
\hline V. Man. Reac. & $\begin{array}{l}1.41 \\
(.49)^{\mathrm{c}}\end{array}$ & $\begin{array}{l}1.60 \\
(.52)^{b}\end{array}$ & $\begin{array}{l}1.73 \\
(.61)^{\mathrm{a}}\end{array}$ & $69.82 * * *$ & .067 & $\begin{array}{l}1.78 \\
(.59)\end{array}$ & $\begin{array}{l}1.39 \\
(.42)\end{array}$ & $284.80 * * *$ & .128 \\
\hline V. Man. Instr. & $\begin{array}{l}1.07 \\
(.21)^{\mathrm{c}}\end{array}$ & $\begin{array}{l}1.12 \\
(.21)^{\mathrm{b}}\end{array}$ & $\begin{array}{l}1.21 \\
(.31)^{\mathrm{a}}\end{array}$ & $37.54 * * *$ & .037 & $\begin{array}{l}1.16 \\
(.27)\end{array}$ & $\begin{array}{l}1.10 \\
(.21)\end{array}$ & $26.27 * * *$ & .013 \\
\hline V. Rela. Reac. & $\begin{array}{l}1.59 \\
(.45)^{\mathrm{c}}\end{array}$ & $\begin{array}{l}1.77 \\
(.44)^{\mathrm{b}}\end{array}$ & $\begin{array}{l}1.90 \\
(.50)^{\mathrm{a}}\end{array}$ & $44.44 * * *$ & .044 & $\begin{array}{l}1.76 \\
(.47)\end{array}$ & $\begin{array}{l}1.77 \\
(.47)\end{array}$ & .018 & .000 \\
\hline V. Rela. Instr. & $\begin{array}{l}1.12 \\
(.27)^{\mathrm{c}}\end{array}$ & $\begin{array}{l}1.17 \\
(.26)^{\mathrm{b}}\end{array}$ & $\begin{array}{l}1.28 \\
(.37)^{\mathrm{a}}\end{array}$ & $31.90 * * *$ & .032 & $\begin{array}{l}1.21 \\
(.32)\end{array}$ & $\begin{array}{l}1.17 \\
(.28)\end{array}$ & $11.84 * *$ & .006 \\
\hline
\end{tabular}

Notas. $\alpha=.05, \mathrm{a}>\mathrm{b}>\mathrm{c}, * * * \mathrm{p}<.001$

V. Man. Reac. = Violencia Manifiesta Reactiva. V. Man. Instr.= Violencia Manifiesta Instrumental. V. Man. Reac. = Violencia Manifiesta Reactiva. V. Man. Instr.= Violencia Manifiesta Instrumental.

\section{Variables demográficas: sexo}

Se obtuvieron diferencias estadísticamente significativas respecto del sexo en las variables violencia manifiesta reactiva, violencia manifiesta instrumental y violencia relacional instrumental. Como se observa en la tabla 2, los chicos, en comparación con las chicas, mostraron puntuaciones más elevadas en violencia manifiesta (rectica e instrumental) y en violencia relacional instrumental.

\section{Análisis de la interacción entre dependencia de las RSV y sexo}

Se obtuvo un efecto de interacción estadísticamente significativo entre dependencia de las RSV, sexo y violencia manifiesta reactiva, $F(5,1946)=92.84, p<.001, \eta^{2}=.193$, violencia manifiesta instrumental, $F(5,1946)=24.78, p<.001, \eta^{2}=.060$, violencia relacional reactiva, $F(5,1946)=22.18$, $p<.001, \eta^{2}=.054$, y violencia relacional instrumental, $F(5,1946)=17.66, p<.001, \eta^{2}=.043$. Los análisis realizados a posteriori indicaron que, los chicos, sobre todo aquellos con alta dependencia de las RSV, se implican en conductas de violencia manifiesta (reactiva e instrumental) con más frecuencia que las chicas (ver Figuras 1 y 2). Sin embargo, cuando los adolescentes participan en actos de violencia relacional reactiva e instrumental, las diferencias entre chicos y chicas son menores 
conforme el grado de dependencia aumenta, de manera que en el grupo de alta dependencia, chicos y chicas mostraron un grado de implicación en estas conductas (ver Figuras 3 y 4).

\section{Figura 1.}

Interacción entre dependencia de las RSV y sexo en violencia manifiesta reactiva.

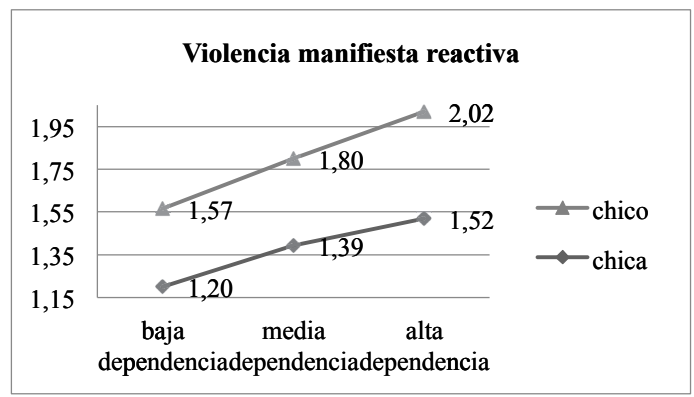

Figura 2.

Interacción entre dependencia de las RSV y sexo en violencia manifiesta instrumental.

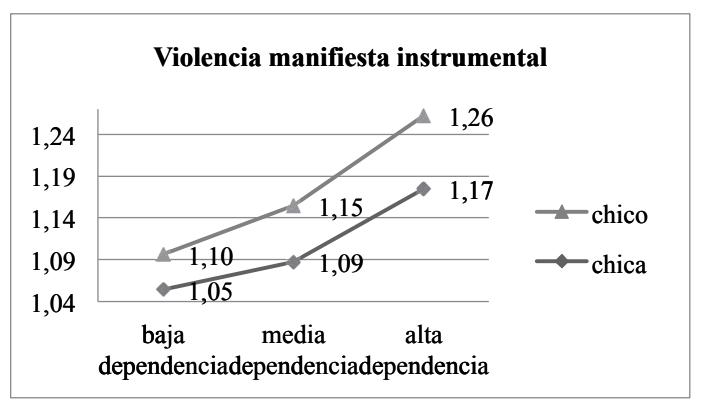

Figura 3.

Interacción entre dependencia de las RSV y sexo en violencia relacional reactiva.

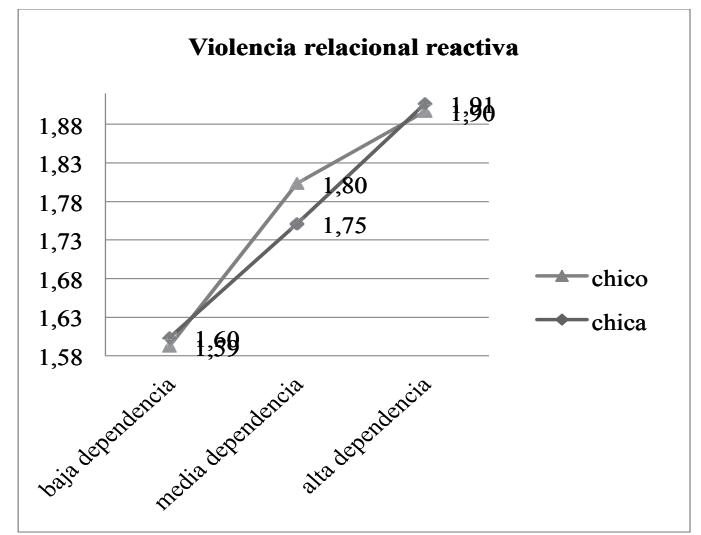


Figura 4.

Interacción entre dependencia de las RSV y sexo en Violencia manifiesta instrumental.

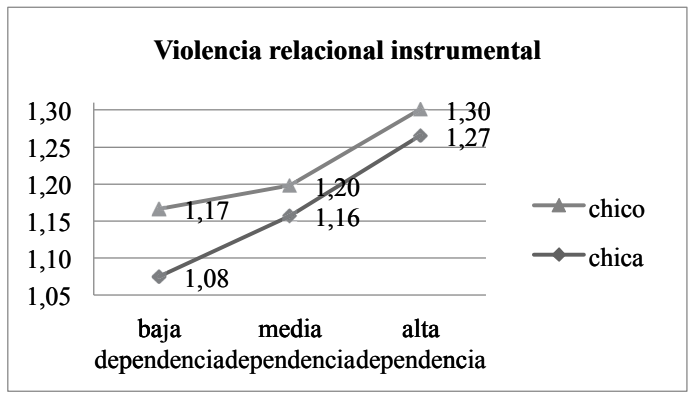

\section{DISCUSIÓN}

El objetivo del presente estudio fue analizar las relaciones existentes entre la dependencia de las RSV y la violencia entre iguales en adolescentes, considerando, además, el sexo y la edad. Los resultados obtenidos permiten confirmar que el grado de dependencia a las RSV se asocia con una mayor implicación en conductas violentas entre iguales. Asímismo, se ha constatado un efecto de interacción entre el sexo y la dependencia, en la violencia manifiesta y en la relacional, tanto reactiva como instrumental.

Los análisis previos realizados han mostrado que las adolescentes muestran una mayor dependencia de las RSV que los chicos, un resultado convergente con los obtenidos por otros autores, en Ios que se concluye que las chicas utilizan con más frecuencia las RSV (Bringué y Sádaba, 2009; Sabater y Bingen, 2015). Estas diferencias pueden explicarse teniendo en cuenta las diferencias de género constatadas en estudios previos respecto del uso de Internet. En este sentido, las adolescentes parecen utilizanen mayor medida que los chicos aquellas herramientas de internet destinadas, principalmente, a la comunicación, al fortalecimiento de las amistades y a la interacción social, pese a que chicos y chicas están apuntados a un número equivalente de plataformas virtuales (Espinar Ruiz y González Río, 2009). Creemos que estos resultados son interesantes puesto que nos indican que las adolescentes constituyen un grupo de mayor vulnerabilidad respecto de la dependencia de las RSV.

Con respecto a los efectos principales, los resultados obtenidos mostraron el efecto de la dependencia de las RSV en la expresión de conductas violentas entre iguales en la adolescencia. Los adolescentes con una mayor dependencia mostraron una mayor implicación en conductas de violencia manifiesta y relacional -reactiva e instrumental- . Estos resultados van en la línea de los obtenidos por Arnáiz et al. (2016), en los que se ha observado el vínculo entre ciberadicción y el bullying.No obstante, los resultados del presente estudio muestran un análisis más profundo de estas relaciones, en la medida en que se observa que conforme mayor es la dependencia de las RSV mayor es la expresión de conductas violentas en la escuela hacia los iguales.

Aunque este resultado resulta sugerente, debido a la escasez de trabajos en los que se analiza la relación entre dependencia de las RSV y violencia entre iguales, creemos que la principal aportación del presente estudio es el análisis de la interacción entre dependencia y sexo respecto de la conducta violenta. Así, de los resultados obtenidos se desprende que las relaciones entre dependencia género y violencia escolar difieren en función del tipo de conducta. Mientras que los chicos se impli- 
can en mayor medida que las chicas en conductas de violencia manifiesta, especialmente cuando la dependencia a las RSV es elevada, estas diferencias tienden a desaparecer en la violencia relacional, especialmente en la reactiva. De manera que, a diferencia de lo que se ha observado en la violencia manifiesta, los chicos con baja dependencia participan en mayor medida en conductas de violencia relacional (reactiva e instrumental) y, además, conforme la dependencia de las RSV aumenta, las diferencias entre sexos en la expresión de estas conductas tienden a desaparecer.

Creemos que estos resultados pueden atribuirse al hecho de que, tradicionalmente, los chicos participan en mayor medida que las chicas en conductas de violencia manifiesta (como pegar, insultar 0 intimidar, entre otros) y que esta tendencia se mantiene estable independientemente de la dependencia a las RSV. Sin embargo, los resultados relativos a participación en conductas de vioIencia relacional en chicas son todavía poco concluyentes. En este sentido, y respecto de la violencia relacional, los datos indican que los chicos participan en mayor medida que las chicas en conductas de violencia relacional instrumental. Sin embargo, cuando se tiene en cuenta la dependencia hacia las RSV, observamos que tanto los chicos como las chicas con alta dependencia utilizanse implican en ambos tipos de violencia relacional de modo parejo. Todo ello parece apuntar a que los chicos y chicas con alta dependencia expresan una mayor violencia relacional hacia sus iguales y que, posiblemente, también se impliquen en estas conductas en las plataformas virtuales. En este sentido, Arnáiz et al. (2016) concluyen que la ciberadicción, el bullying y el cyberbullying se encuentran interrelacionados. No obstante, creemos que esta relación debería examinarse en futuras investigaciones.

Este estudio cuenta con aspectos positivos pero también con limitaciones. Entre los aspectos positivos cabe destacar el hecho de que es un ámbito de estudio novedoso, en el que apenas existe evidencia empírica. En este sentido los resultados del presente estudio pretenden contribuir a un mayor conocimiento científico en un ámbito poco explorado. También, un aspecto que, a nuestro juicio, constituye la principal aportación del presente estudio, es el efecto de interacción de dependencia a las RSV y sexo. Este resultado sugiere la necesidad de incorporar una perspectiva de género en futuras investigaciones en las que se explora la continuidad entre conductas problemáticas en los ámbitos virtual y no virtual en adolescentes. Entre las limitaciones, cabe mencionar que la naturaleza transversal del estudio no permite establecer relaciones causales entre las variables estudiadas, por lo que se sugiere un diseño longitudinal que permita profundizar en estas relaciones. Además, todas las fuentes provienen de autoinforme, lo cual puede implicar sesgos, especialmente en temas sensibles como la violencia y el uso abusivo de las RSV.

Pese a estas limitaciones, los resultados del presente muestran que las adolescentes, a diferencia de lo que ocurre en otras etapas evolutivas, son más proclives a utilizar de manera abusiva las RSV y que este uso abusivo se asocia con una mayor expresión de conductas violentas en la escuela hacia sus iguales, especialmente de carácter relacional.

\section{REFERENCIAS}

Arab, L. \& Díaz, G. (2015). Impacto de las redes sociales e internet en la adolescencia: aspectos positivos y negativos. Revista Médica Clínica Las Condes, 26(1), 7-13. doi: 10.1016/j.rmclc.2014.12.001

Arnaiz, P., Cerezo, F., Gimenez, A. \& Maquilón, J. (2016). Conductas de ciberadicción y experiencias de cyberbullying entre adolescentes. Anales De Psicología, 32(3), 761. http://dx.doi.org/10.6018/analesps.32.3.217461

Becoña, E. (2006). Adicción a nuevas tecnologías. Vigo: Nova Galicia Edicións.

Blanco, M.A. (2014). Implicaciones del uso de las redes sociales en el aumento de la violencia de 
género en adolescentes. Comunicación y Medios, 30, 124-141.

Bringué, X. \&Sádaba, C. (2009). La generación interactiva en España: Niños y adolescentes ante las pantallas. Barcelona y Madrid: Ariel y Fundación Telefónica.

Cao, H., Sun, Y., Wan, Y., Hao, J.\& Tao, F. (2011). Problematic Internet use in Chinese adolescents and its relation to psychosomatic symptoms and life satisfaction. BMC PublicHealth, 11(1), 18. doi: 10.1186/1471-2458-11-802

Catalina García, B., López de Ayala López, M.\& García Jiménez, A. (2014). Los riesgos de los adolescentes en Internet: Ios menores como actores y víctimas de los peligros de Internet. doi: 10.4185/rlcs-2014-1020

Carbonell, X., Chamarro, A., Beranuy, M., Griffiths, M., Oberst, U., Cladellas, R. \&Talarn, A. (2012) Problematic Internet and cell phone use in Spanish teenagers and young students. Anales de Psicología, 28(3) 789-796. doi: 10.6018/analesps.28.3.156061

Carbonell, X., Fúster, H., Chamarro, A.\&Oberst, U. (2012). Adicción a internet y móvil: una revisión de estudios empíricos españoles. Papeles del Psicólogo, 33(2), 82-89.

Castells, M. (2001). Internet y la sociedad red. Barcelona: Alianza.

Chóliz, M. \& Marco, C. (2012). Adicción a Internet y Redes sociales. Tratamiento psicológico. Madrid: Alianza Editorial.

Devine, P. \& Lloyd, K. (2012). Internet use and psychological well-being among 10-year-old and 11year-old children. Child Care in Practice, 18(1), 5-22. doi: 10.3109/09638237.2012.670883

Echeburúa, E. \& De Corral, P. (2010).Adicción a las nuevas tecnologías ya las redes sociales en jóvenes: un nuevo reto. Adicciones, 22(2), 91-96. doi: 10.20882/adicciones.196

Echeburúa, E., Labrador, F.J. \&Becoña, E. (2009). Adicción a las nuevas tecnologías. Madrid: Pirámide.

Erdur-Baker, 0̈. (2010). Cyberbullying and its correlation to traditional bullying, gender and frequent and risky use of internet-mediated communication tools. New Media \&Society, 12(1), 109-125. doi: $10.1177 / 1461444809341260$

Espinar Ruiz, E. \& González Río, M. (2009). Jóvenes en las redes sociales virtuales: un análisis exploratorio de las diferencias de género. Feminismos, (14), 87-105. doi: 10.14198/fem.2009.14.06

Estévez, L., Bayón, C., de la Cruz, J. \& Fernández-Líria, A. (2009). Uso y abuso de Internet en adolescentes. En E. Echeburúa, F.J. Labrador y E. Becoña (eds.). Adicción a las nuevas tecnologías (pp. 101-130). Madrid: Pirámide.

García, A., López de Ayala, M. \& Gaona, C. (2012). La investigación sobre los usos y los riesgos de los menores en el ciberespacio. Análisis metodológico. Doxa Comunicación: revista interdisciplinar de estudios de comunicación y ciencias sociales, 13, 13-41.

Garmendia, M., Garitaonandia, C., Martínez, G. \& Casado, M.A. (2011). Riesgos y seguridad en internet: Los menores españoles en el contexto europeo. Universidad del País Vasco/EuskalHerrikoUnibertsitatea, Bilbao: EU Kids Online. Recuperado de http://www.eukidsonline.net

Giménez, A. M.; Maquilón, J. J.\& Arnaiz, P. (2015). Usos problemáticos y agresivos de las TIC por parte de adolescentes implicados en cyberbullying. Revista de Investigación Educativa, 33(2), 335-351. doi:10.6018/rie.33.2.199841

Hearn, G. \&Foth, M. (2007).Communicative Ecologies. Electronic Journal ofCommunication, 17 (12). Recuperado: de http://www.cios.org/www/ejc/v17n12.htm\#PréfaceEditoriale

Instituto Nacional de Estadística (INE) (2012). Encuesta sobre equipamiento y uso de tecnologías de la información y comunicación en los hogares, Recuperado de http://www.ine.es/metodolo- 
gia/t25/t25304506613.pdf

Johansson, A. \&Götestam, K.G. (2004). Internet addiction: characteristics of a questionnaire and prevalence in Norwegian youth (12-18 years). Scandinavian Journal of Psychology, 45, 223229. doi: 10.1111/j.1467-9450.2004.00398.x

Little, T. D., Henrich, C. C., Jones, S. M. \& Hawley, P. H. (2003).Disentangling the "whys" from the "whats" of aggressive behaviour. International Journal of Behavioral Development, 27, 122- 133.

Martín, A., Pazos, M., Montilla, M. \& Romero, C. (2016). Una modalidad actual de violencia de género en parejas de jóvenes: las redes sociales. Educación XX1, 19(2), 405-429. http://dx.doi.org/10.5944/educxx1.16473

Muñoz-Rivas, M. J., Navarro, M .E. \& Ortega, N. (2003). Patrones de uso de Internet en población universitaria española. Adicciones, 15, 137-144. doi: 10.20882/adicciones.437

Muñoz-Rivas, M. J., Fernández, L. \& Gámez-Guadix, M. (2010). Analysis of the indicators of pathological Internet use in Spanish university students. The Spanish Journal of Psychology, 13 (2),697-707.

Pedrero, E. J., Rodríguez, M. T. \& Ruiz, J. M. (2012).Adicción o abuso del teléfono móvil. Revisión de la literatura. Adicciones, 24(2), 139-152. doi: 10.20882/adicciones.107

Rial, A., Gómez, P., Braña, T. \& Varela, J. (2014). Actitudes, percepciones y uso de Internet y las redes sociales entre los adolescentes de la comunidad gallega (España). Anales de Psicología, 30(2), 642-655. doi: 10.6018/analesps.30.2.159111

Rodríguez, A. \& Fernández, A. P. (2014). Relación entre el tiempo de uso de las redes sociales en internet y la salud mental en adolescentes colombianos. Acta Colombiana de Psicología, 17(1), 131-140. doi: 10.14718/ACP.2014.17.1.13

Sabater, C. \&Bingen, J. (2015). No sin mi móvil. Diferencias de género y uso de las nuevas tecnologías. Icono, 14(13), 208-246. doi: 10.7195/ri14.v13i1.722 